\title{
Amplitude of the magnetic anomaly vector in low latitudes via equivalent layer
}

\author{
Shayane P. Gonzalez*, Felipe F. Melo, Valéria C. F. Barbosa and Vanderlei C. Oliveira Junior, Observatório Nacional
}

Copyright 2019, SBGf - Sociedade Brasileira de Geofísica

This paper was prepared for presentation during the $16^{\text {th }}$ International Congress of the Brazilian Geophysical Society held in Rio de Janeiro, Brazil, 19-22 August 2019.

Contents of this paper were reviewed by the Technical Committee of the $16^{\text {th }}$ International Congress of the Brazilian Geophysical Society and do not necessarily represent any position of the SBGf, its officers or members. Electronic reproduction or paper for commercial purposes without the written consent of the Brazilian Geophysical Society is prohibited.

\section{Abstract}

The interpretation of total-field anomalies data presents complexities due to dipolar characteristics of the anomalies. Usually, the geophysicists apply some transformations to the measured total-field anomaly to assist the magnetic interpretation and inversion. However, it can lead to an unstable transformed data at low latitude regions. To transform the total-field anomaly into the amplitude of the magnetic anomaly vector, we can use the equivalent layer technique and the Fourier filtering. These two methods are applied to synthetic magnetic data simulated at high- and low-magnetic latitudes. At high latitude, the computed amplitude of the magnetic anomaly vector either by applying the Fourier filtering or by employing the equivalent layer technique is equal to the true one. However, at low latitudes, the computed amplitude of the magnetic anomaly vector differs from the true one either by applying the Fourier filtering or by employing the equivalent layer technique. Both computed amplitudes of the magnetic anomaly vector exhibit striations in the direction of declination at low latitudes. However, at low latitudes, the magnitudes of the differences between the true and the computed amplitude of the magnetic anomaly vector produced by employing the equivalent layer technique are substantially smaller than the ones produced by applying the Fourier filtering. The better result produced by the equivalent layer technique is because the stabilization imposed by the Tikhonov regularization. We also applied the equivalent layer technique and the Fourier filtering to real total-field anomaly at a low latitude region encompassing the Central Amazonian Province and the Carajás Mineral Province, Brazil, aiming at computing the amplitude of the magnetic anomaly vector. Likewise, both transformations exhibit undesirable striations; however the one obtained via the equivalent layer technique yields better result in comparison with those obtained via the Fourier filtering.

\section{Introduction}

The magnetic method has been used as an aid to exploration of mineral deposits over the last decades. However, the observed magnetic anomalies are generally asymmetrical and have a dipolar shape, which makes the data interpretation difficult. To assist the geologic interpretation or inversion, some potential-field transformations can be applied to the measured data (Blakely, 1996). The total-field anomalies can be transformed, for example, into the three orthogonal components of the magnetic field. From these components is possible to compute the amplitude of the magnetic anomaly vector.

In cases of remanent magnetization at low latitudes, the amplitude of the magnetic anomaly vector can be used as input for inversion, since it does not critically depend on the knowledge of the magnetization direction of the bodies (Li et al. 2010; Leão-Santos et al., 2015). In addition, Krahenbuhl and Li (2017) used the amplitude of the magnetic anomaly vector as input for inversion in highly magnetic environments under strong selfdemagnetization.

In this work, we compare two methods to compute the amplitude of the magnetic anomaly vector at high and low latitudes: the Fourier filtering (Pedersen, 1978; Blakely, 1996) and the equivalent layer technique (Dampney, 1969; Siqueira et al. 2017). The transformation of the total-field anomaly into the amplitude of the magnetic anomaly vector via Fourier filtering consists in multiplying the total-field anomaly in Fourier domain by a filter (Blakely, 1996). On the other hand, the same transformation via the equivalent layer technique requires the solution of large linear system of equations in space domain, which usually demands a costly computation time. We stress that, at low latitudes, the computed amplitudes of the magnetic anomaly vector calculated either by Fourier filtering or by equivalent layer technique are unstable. Specifically, at low latitudes, the transformed data exhibits undesired striations (elongated signals) in the declination direction. Because the equivalent layer technique stabilizes the solution by using the Tikhonov regularization, the amplitude of the magnetic anomaly vector is better obtained by the equivalent layer technique. Specifically, at low latitudes, the magnitudes of the striations in the amplitude of the magnetic anomaly vector obtained by equivalent layer technique are smaller than those obtained by Fourier filtering, likewise observed in the reduction to the pole by Silva (1986). This conclusion has been confirmed in tests with synthetic data and in the case of total-field anomaly at a low latitude region encompassing the Central Amazonian Province and the Carajás Mineral Province, located at Pará state, Brazil.

\section{Method}

The amplitude of the magnetic anomaly vector $\mathbf{B}$ is given by:

$$
|B|=\sqrt{\mathbf{B}_{\mathbf{x}}^{2}+\mathbf{B}_{\mathbf{y}}^{2}+\mathbf{B}_{\mathbf{z}}^{2}}
$$

where $\mathbf{B}_{\mathbf{x}}, \mathbf{B}_{\mathbf{y}}$ e $\mathbf{B}_{\mathbf{z}}$ are the three components of the magnetic anomaly vector in the $x$-, $y$ - and $z$-directions, that points to north, east and downward, respectively. The three orthogonal components can be obtained from the 
total-field anomaly by using the Fourier domain filtering (Pedersen, 1978; Blakely, 1996) or by the equivalent source technique (Dampney, 1969). As a benchmark for comparison of the methods, the components of the magnetic anomaly vector were computed based on the subroutine B.3 published by Blakely (1996) and the true amplitude of the magnetic anomaly vector was computed from equation 1 .

\section{Amplitude of the magnetic anomaly vector via Fourier filtering}

The total-field anomaly $\Delta \mathbf{T}$ can be transformed into the $\mathbf{B}_{\boldsymbol{\alpha}}$-component of the magnetic anomaly vector, where $\alpha$ belongs to the set of $x-, y$-, and $z$-directions of a rightsided Cartesian coordinate system, according to the follow equation (Blakely, 1996):

$$
\mathcal{F}\left\{\mathbf{B}_{\alpha}\right\}=\mathcal{F}\{\boldsymbol{\Delta} \mathbf{T}\} \mathcal{F}\left\{\psi_{\alpha}\right\} .
$$

The filter $\mathcal{F}\left\{\psi_{\alpha}\right\}$ is given by

$$
\begin{aligned}
\mathcal{F}\left\{\psi_{x}\right\} & =\frac{j k_{x}}{|k| \hat{f}_{z}+j\left(\hat{f}_{x} k_{x}+\hat{f}_{y} k_{y}\right)}, \\
\mathcal{F}\left\{\psi_{y}\right\} & =\frac{j k_{y}}{|k| \hat{f}_{z}+j\left(\hat{f}_{x} k_{x}+\hat{f}_{y} k_{y}\right)},
\end{aligned}
$$

and

$$
\mathcal{F}\left\{\psi_{z}\right\}=\frac{1}{|k| \hat{f}_{z}+j\left(\hat{f}_{x} k_{x}+\hat{f}_{y} k_{y}\right)},
$$

where $j=\sqrt{-1}, k$ is the angular wavenumber and $k_{x}$ and $k_{y}$ are the wavenumbers in the $x$ - and $y$-directions. The unit vector $\hat{\mathbf{F}}=\left(\hat{f}_{x}, \hat{f}_{y}, \hat{f}_{z}\right)$ is defined by:

$$
\hat{\mathbf{F}}=\left[\begin{array}{cc}
\cos I_{0} & \cos D_{0} \\
\cos I_{0} & \sin D_{0} \\
\sin I_{0}
\end{array}\right],
$$

where $I_{0}$ and $D_{0}$ are the inclination and declination of the main geomagnetic field at the study area. The knowledge about the direction of the main field is sufficient to transform the total-field anomaly, as shown in equations 3-6. However, this kind of transformation will be unstable at low-latitudes (Blakely, 1996).

Amplitude of the magnetic anomaly vector via equivalent layer technique

A discrete set of total-field anomaly data can be expressed by the transposed vector $\mathbf{d}^{\mathbf{0}}=\left[d_{1}^{o}, \cdots, d_{N}^{o}\right]^{\mathrm{T}}$, where each element $d_{i}^{o}$ depicts an observation at the point $\left(x_{i}, y_{i}, z_{i}\right), i=1, \cdots, N$. Let $\mathbf{d}(\mathbf{p})=\left[d_{1}, \cdots, d_{N}\right]^{\mathrm{T}}$ be the predicted total-field anomaly vector, being its $i$ th term expressed by:

$$
d_{i}(\boldsymbol{p})=\sum_{l=1}^{M} p_{l} g_{i l},
$$

where $p_{l}$ is the magnetic moment intensity (in $\mathrm{Am}^{2}$ ) associated to the $l$ th equivalent source (e.g., dipole). The harmonic function $g_{i l}$ represents the projection onto the main field of the magnetic induction produced, at the observation point $\left(x_{i}, y_{i}, z_{i}\right)$, by a dipole with unit magnetic moment, located at a point $\left(x_{l}, y_{l}, z_{l}\right)$ :

$$
g_{i l}=c_{m} \frac{\mu_{0}}{4 \pi} \hat{\mathbf{F}}^{\mathrm{T}} \mathbf{H}_{\mathbf{i l}} \hat{\mathbf{h}},
$$

where $c_{m}=10^{9}$ is a constant factor transforming the magnetic induction from Tesla $(\mathrm{T})$ to nanotesla (nT), $\mu_{0}=4 \pi \times 10^{-7} \mathrm{H} / \mathrm{m}$ is the magnetic constant and $\hat{\mathbf{F}}$ is given by equation 6 . The unit vector $\hat{\mathbf{h}}$ is given by:

$$
\hat{\mathbf{h}}=\left[\begin{array}{c}
\cos I \cos D \\
\cos I \sin D \\
\sin I
\end{array}\right],
$$

where $I$ and $D$ are the inclination and declination of the magnetized equivalent source. The matrix $\mathbf{H}_{\mathbf{i l}}$ is given by the second derivatives of the inverse distance function $\left(r^{-1}=\left[\left(x_{i}-x_{l}\right)^{2}+\left(y_{i}-y_{l}\right)^{2}+\left(z_{i}-z_{l}\right)^{2}\right]^{-1 / 2}\right)$. Notice that equation 7 can be expressed in matrix form:

$$
\mathbf{d}(\mathbf{p})=\mathbf{G} \mathbf{p} \text {, }
$$

where $\mathbf{G}$ is an $N \times M$ sensitivity matrix that contains the elements $g_{i l}$ and $\mathbf{p}$ is an $M \times 1$ vector of parameters, whose $l$ th element is the coefficient $p_{j}$ associated with the $l$ th equivalent source in equation 7 .

A stable estimate of the vector of parameters $\mathbf{p}^{*}$ can be obtained by minimizing the residuals, that is the difference between the observed $\mathbf{d}^{\mathbf{o}}$ and the predicted data $\mathbf{d}(\mathbf{p})$. Usually, a regularization function is used in this inversion (Tikhonov and Arsenin, 1977; Aster et al., 2005). After obtaining the vector $\mathbf{p}^{*}$, is possible to transform the totalfield anomaly into a desired $\mathbf{B}_{\boldsymbol{\alpha}}$-component of the magnetic anomaly vector $\mathbf{B}$, i.e.:

$$
\mathbf{B}_{\boldsymbol{\alpha}}=\mathbf{T}^{\alpha} \mathbf{p}^{*},
$$

where $\mathbf{T}^{\boldsymbol{\alpha}}$ is an $N \times M$ matrix of transformation, which elements are given by

$$
t_{i l}^{\alpha}=c_{m} \frac{\mu_{0}}{4 \pi} \mathbf{H}_{\mathrm{il}}^{\alpha} \hat{\mathbf{h}},
$$

where $\mathbf{H}_{\mathrm{il}}^{\alpha}$ is the $\boldsymbol{\alpha}$ th row of matrix $\mathbf{H}_{\mathrm{il}}$.

\section{Synthetic tests}

We performed three synthetic tests simulating total-field anomalies generated by a sphere with a constant magnetization intensity of $5 \mathrm{~A} / \mathrm{m}$. The magnetic source has $500 \mathrm{~m}$ of radius and is centered on $x=y=0 \mathrm{~km}$ and $z=2 \mathrm{~km}$. The anomalies were calculated on a regular grid of $70 \times 70$ observation points and the acquisition flight height is $-150 \mathrm{~m}$. The observed data were corrupted with $1 \%$ of pseudorandom noise.

In the first test, the main field has inclination $I_{0}=60^{\circ}$ and declination $D_{0}=-20^{\circ}$ simulating a field at high latitude. The source (outlined by a black circle in Figure 1) has only induced magnetization, that is $\hat{\mathbf{F}}$ and $\hat{\mathbf{h}}$ are equal $\left(I_{0}=I ; D_{0}=D\right)$. Figure $1 \mathrm{a}$ and $1 \mathrm{~b}$ shows the total-field anomaly and the true amplitude of the magnetic anomaly vector. Figure $1 \mathrm{c}$ and $1 \mathrm{e}$ presents the amplitude of the magnetic anomaly vector obtained via Fourier filtering and equivalent layer technique, respectively. In this highlatitude scenario, both estimates provide a high degree of similarity with the true amplitude data. We stress that the maxima of the amplitudes of the magnetic anomaly vector (Figure 1c and 1e) locate the horizontal position of the simulated source (outlined by a black circle). Moreover, the intensity of the amplitudes of the magnetic anomaly vector (Figure 1c and 1e) decays softly and no artifact is generated. Figure $1 \mathrm{~d}$ and $1 \mathrm{f}$ shows the residuals (the 
differences between the true and the computed amplitudes of the magnetic anomaly vector) generated using Fourier filtering and the equivalent layer technique, respectively. The amplitudes of the residuals are negligible when compared to the forward model, confirming the fit of the data.
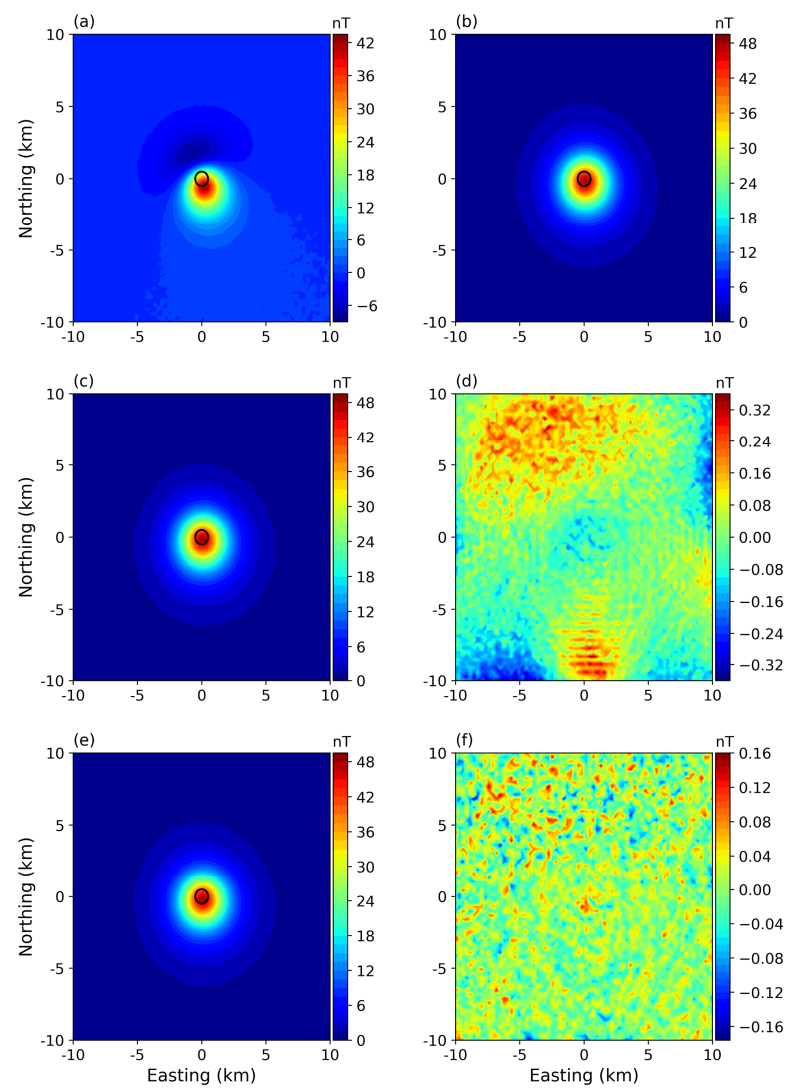

Figure 1- Synthetic test 1: field at high latitude produced by a source with induced magnetization. The black circle outlines the source. The main field and source magnetization are $I_{0}=I=$ $60^{\circ}$ and $D_{0}=D=-20^{\circ}$. (a) Total-field anomaly. (b) True amplitude of the magnetic anomaly vector. (c) Amplitude of the magnetic anomaly vector computed in Fourier domain. (d) Residuals defined as the differences between the true and the computed amplitudes of the magnetic anomaly vector, the latter is obtained in Fourier domain. (e) Amplitude of the magnetic anomaly vector computed via equivalent layer technique. (f) Residuals defined as the differences between the true and the computed amplitudes of the magnetic anomaly vector, the latter is obtained by the equivalent layer technique.

In the second test, the main field has $I_{0}=-8^{\circ}$ and $D_{0}=-20^{\circ}$ simulating a field at a low latitude. The source (outlined by a black circle in Figure 2) has purely induced magnetization $\left(I_{0}=I ; D_{0}=D\right)$. Figure $2 \mathrm{a}$ and $2 \mathrm{~b}$ shows the total-field anomaly and the true amplitude of the magnetic anomaly vector, respectively. Figure $2 c$ and $2 e$ shows the amplitudes of the magnetic anomaly vector computed by Fourier filtering and equivalent layer technique, respectively. Notice that both computed amplitudes of the magnetic anomaly vector (Figure $2 \mathrm{c}$ and $2 e$ ) exhibit high frequency artifacts elongated in the direction of declination because this transformation was performed at low latitudes. However, the computed amplitude of the magnetic anomaly vector (Figure $2 \mathrm{e}$ ) via equivalent layer technique at low latitude is more stable than the one obtained with the Fourier filtering (Figure $2 c$ ). Figure $2 d$ and $2 f$ shows the residuals defined as the differences between the true and the computed amplitudes of the magnetic anomaly vector produced by employing the Fourier filtering and equivalent layer technique, respectively. Based on the small magnitudes of both residual maps (Figure $2 \mathrm{~d}$ and $2 \mathrm{f}$ ) we can say that both methods (Fourier filtering and equivalent layer technique) yield good computed amplitudes of the magnetic anomaly vector (Figure $2 \mathrm{c}$ and $2 \mathrm{e}$ ). However, we stress that the residuals with equivalent layer technique (Figure 2f) are smaller than those obtained with Fourier filtering (Figure 2d) and they are symmetrically distributed around zero. Hence, the computed amplitude of the magnetic anomaly vector using equivalent layer technique (Figure 2e) is more stable than the one computed by Fourier filtering (Figure 2c).
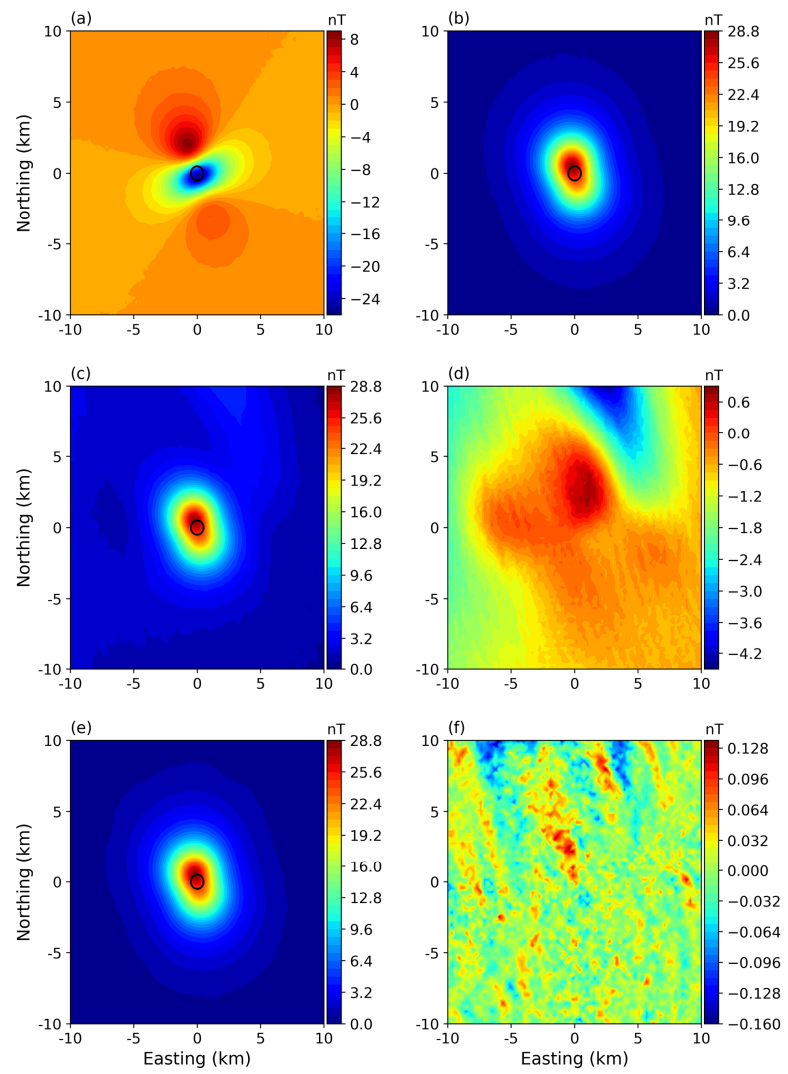

Figure 2- Synthetic test 2: field at low latitude produced by a source with induced magnetization. The black circle outlines the source. The main field and source magnetization are $I_{0}=I=$ $-8^{\circ}$ and $D_{0}=D=-20^{\circ}$. (a) Total-field anomaly. (b) True amplitude of the magnetic anomaly vector. (c) Amplitude of the magnetic anomaly vector computed in Fourier domain. (d) Residuals defined as the differences between the true and the computed amplitudes of the magnetic anomaly vector, the latter is obtained in Fourier domain. (e) Amplitude of the magnetic anomaly vector computed via equivalent layer technique. (f) Residuals defined as the differences between the true and the computed amplitudes of the magnetic anomaly vector, the latter is obtained by the equivalent layer technique. 
In the last test, the main field has $I_{0}=-8^{\circ}, D_{0}=-20^{\circ}$ and the sphere has $I=-45^{\circ}$ and $D=-30^{\circ}$, simulating an anomaly at a low latitude generated by a source (outlined by a black circle in Figure 3 ) with remanent magnetization.
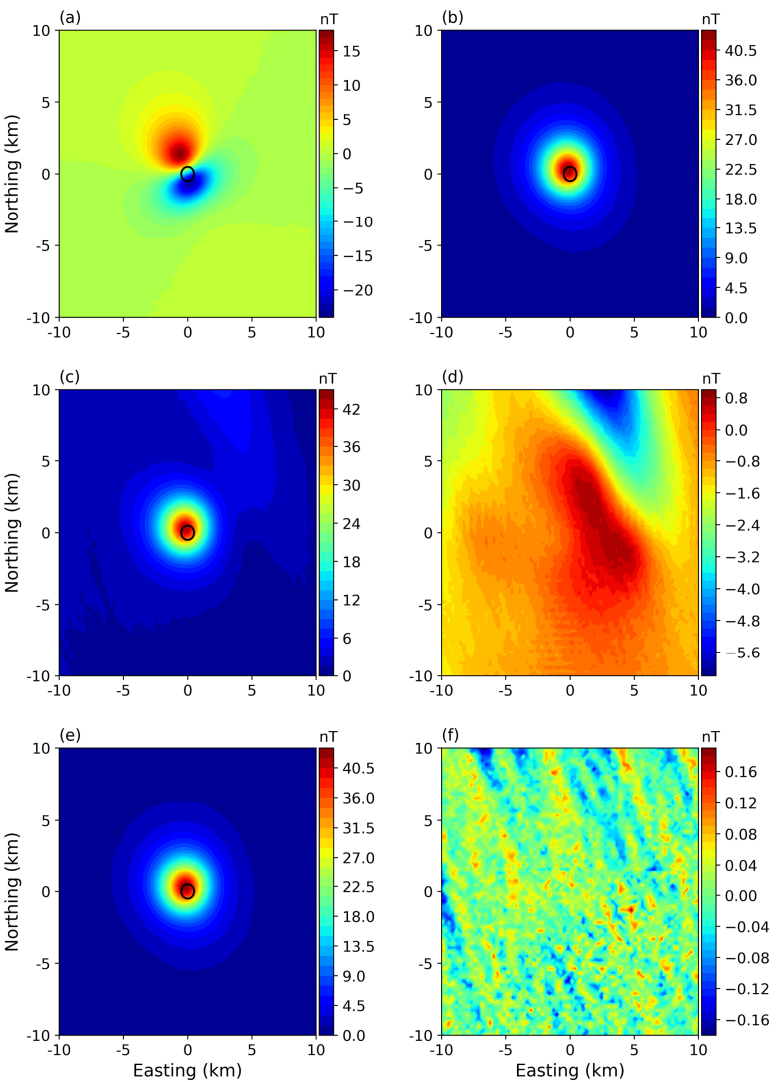

Figure 3- Synthetic test 3: field at low latitude produced by a source with remanent magnetization. The black circle outlines the source. The main field magnetization is $I_{0}=-8^{\circ}$ and $D_{0}=20^{\circ}$; the source magnetization is $I=-45^{\circ}$ and $D=-30^{\circ}$. (a) Total-field anomaly. (b) True amplitude of the magnetic anomaly vector. (c) Amplitude of the magnetic anomaly vector computed in Fourier domain. (d) Residuals defined as the differences between the true and the computed amplitudes of the magnetic anomaly vector, the latter is obtained in Fourier domain. (e) Amplitude of the magnetic anomaly vector computed via equivalent layer technique. (f) Residuals defined as the differences between the true and the computed amplitudes of the magnetic anomaly vector obtained by the equivalent layer technique.

Figure $3 a$ and $3 b$ shows the corresponding total-field anomaly and the true amplitude of the magnetic anomaly vector, respectively. Both transformed amplitudes of the magnetic anomaly vector with Fourier filtering (Figure 3c) and with equivalent layer technique (Figure $3 e$ ) present elongated artifacts. Although these methods yield good transformations, they are clearly unstable at low-latitudes areas. For this low-latitudes scenario, as in the previous test, the computed amplitude of the magnetic anomaly vector via equivalent layer technique (Figure $3 e$ ) yields a better result when compared with the benchmark in Figure $3 \mathrm{~b}$. The comparison between the residuals generated by both methods (Figure $3 d$ and $3 f$ ) corroborates the advantage of using the equivalent layer technique. The residuals from the Fourier filtering (Figure 3d) present higher amplitudes and more prominent elongated artifacts than the residuals calculated from equivalent layer technique (Figure 3f).

\section{Application to real dataset}

Our study area is located in two provinces: the Central Amazonian Province (CAP) and the Carajás Mineral Province (CMP). The Central Amazonian Province is composed by the oldest continental crust of the Amazonian craton (Tassinari and Macambira, 1999). According to Klein et al. (2016) this region comprises widespread volcano-plutonic associations, continental sedimentary covers, and small and sparse (unmapped) basement inliers. The Carajás Mineral Province is the major Archean crustal segment of the Amazonian craton (Almeida et al., 2010). It is a highly mineralized metallogenic province in the northern region of Brazil, in Pará state, known for its deposits of gold, copper, iron and manganese, among others (Grainger et al., 2008). We focus our study on the region that encompasses the east part of the CAP, in the Iriri-Xingu Domain, and in the west part of the CMP, in the Rio Maria domain.

The real aeromagnetic data were acquired between 2013 and 2014 (CPRM, 2015) over the Pará state. The flight lines in the north-south direction were acquired every $3000 \mathrm{~m}$, the tie lines were acquired every $12 \mathrm{~km}$ and the flight height was approximately constant at $900 \mathrm{~m}$. The data set is gridded with the same size in the $x$ - and $y$ directions, $750 \mathrm{~m}$. Figure 4 shows the Carajás survey in yellow, the study area in blue, the black star points to Serra Pelada mine and the inset shows the location of the survey in Brazil.

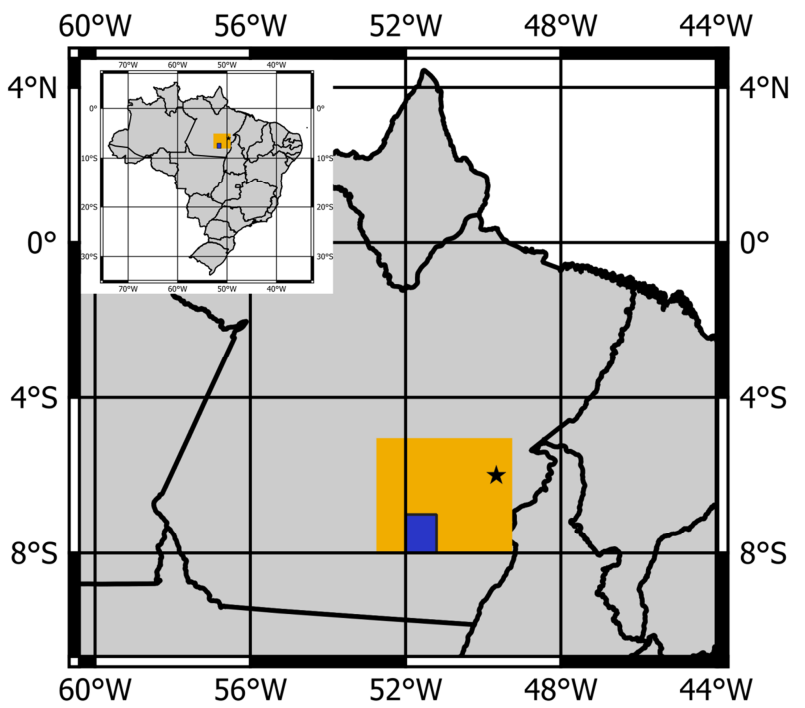

Figure 4- The Carajás survey is in yellow, the study area is in blue, the black star points to Serra Pelada mine and the inset shows the location of the survey in Brazil.

Figure 5 a shows the total-field anomaly of the study area. The main part of the study area is located in the Iriri-Xingu Domain, in the CAP, surrounded by the Rio Maria Domain, in the CMP. The presence of an anomaly with 
dipolar shape is remarkable in the southeast part of the region. The values of inclination $I_{0}=-8^{\circ}$ and declination $D_{0}=-20^{\circ}$ applied to calculate the amplitude of the magnetic anomaly vector, using Fourier filtering and equivalent layer technique, were computed from Chulliat et al. (2014). Moreover, we assume that the sources present only induced magnetization $\left(I_{0}=I=-8^{\circ}\right.$ and $D_{0}=D=-20^{\circ}$ ). We set a planar equivalent layer $4400 \mathrm{~m}$ deep from the surface, composed by $146 \times 106$ dipoles, each one directly beneath an observation point. The zero-order Tikhnov regularization was applied to conditionate the linear system.
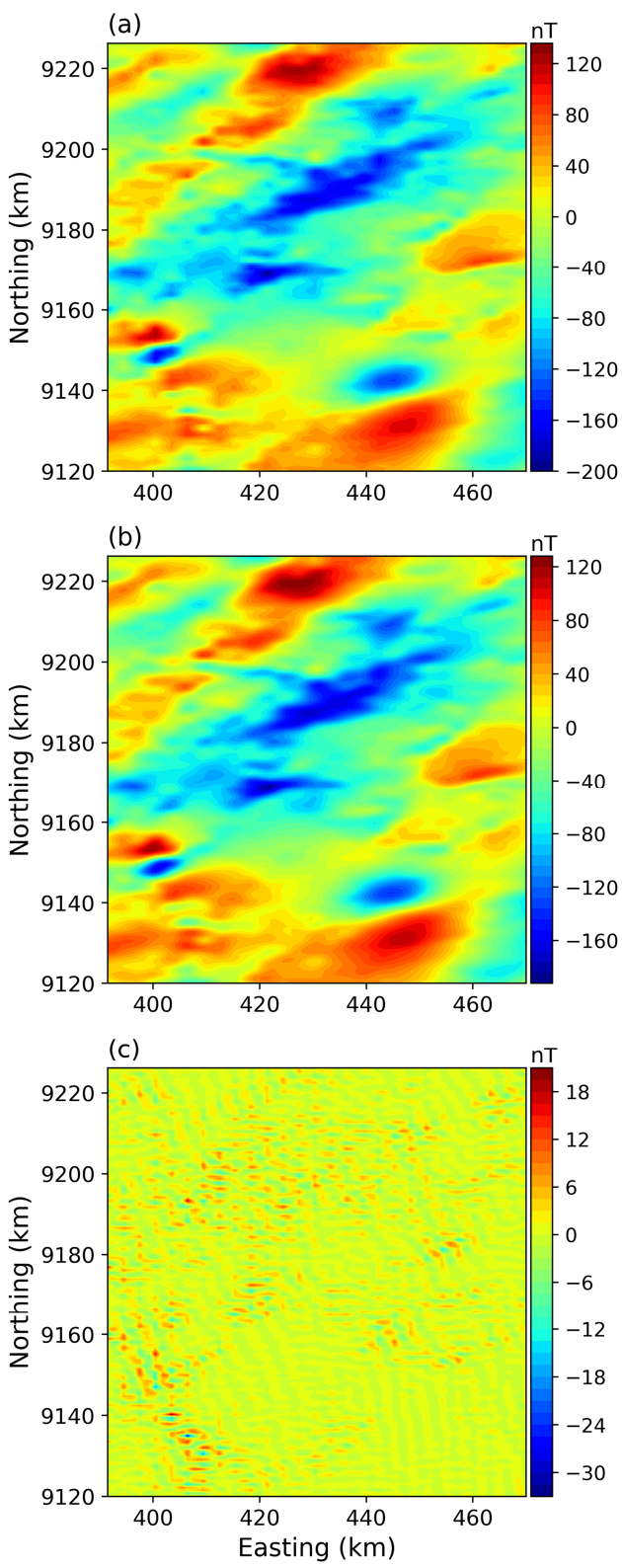

Figure 5- Real dataset application. (a) Total-field anomaly in the southern part of the Rio Maria domain, at the Carajás Mineral Province, Brazil. (b) Predicted total-field anomaly via the equivalent layer technique. (c) Residuals defined as the differences between the observed and the predicted total-field anomalies; the latter is obtained via equivalent layer technique.
To verify the validity of the equivalent layer technique in low latitudes, we show the predicted total-field anomaly (Figure $5 b$ ) and the residuals (Figure $5 c$ ). The similarity between the observed and the predict data is remarkable, the differences are small and coherent residuals are verified. Therefore, we use the estimated parameters (not shown) to compute the amplitude of the magnetic anomaly vector.

Figure $6 a$ shows the transformed amplitude of the magnetic anomaly vector using Fourier filtering. The results show elongated artifacts in the direction of declination and random high frequency artifacts, confirming that the Fourier filtering yields unstable amplitude of the magnetic anomaly vector at low magnetic latitudes. Conversely, the predicted amplitude of the magnetic anomaly vector via equivalent layer technique (Figure 6b) does not present noteworthy artifacts. Notice in the south part of the study area that the maximum peak of amplitude of the magnetic anomaly vector (Figure 6b) coincides with the dipolar signal of the observed total-field anomaly (Figure $5 \mathrm{a}$ ). Other possible sources can be located in the north and southwest of the study area. In this way, the equivalent sources technique overcomes the problems related to magnetic anomalies at low latitudes and opens up the possibility of geologic interpretations.
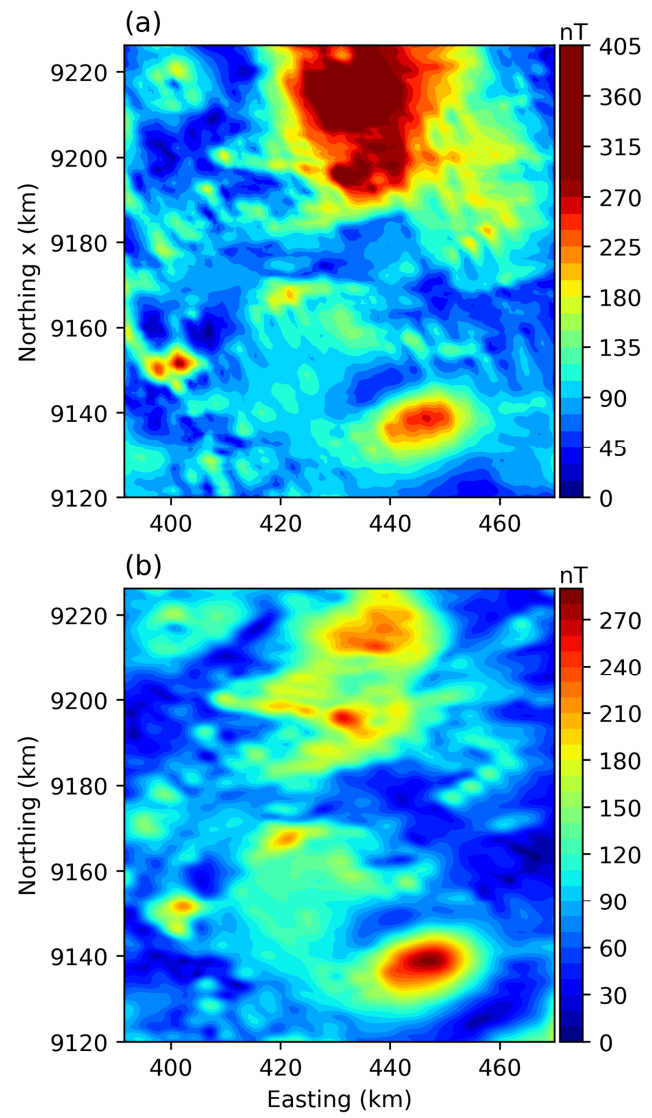

Figure 6- Real dataset application - Computed amplitudes of the magnetic anomaly vector obtained from observed total-field anomaly (figure 5a) via: (a) Fourier filtering and (b) equivalent layer technique. 


\section{Conclusions}

We exemplify the transformation of the total-field anomaly into the amplitude of the magnetic anomaly vector at highand low-latitude regions. The transformed data is obtained by using two methods: Fourier filtering and equivalent layer technique. Synthetic tests show that both methods yield excellent amplitudes of the magnetic anomaly vector at high latitudes. At low latitudes, both methods yield good amplitudes of the magnetic anomaly vector, however they are unstable. Specifically, the computed amplitudes of the magnetic anomaly vector exhibit artifacts and striations in the direction of declination. However, at low latitudes, the obtained amplitude of the magnetic anomaly vector with equivalent layer technique is more stable than one obtained with the Fourier filtering. The application to real dataset encompassing the Central Amazonian Province and the Carajás Mineral Province, Brazil, also shows that the equivalent layer technique is more effective at low inclinations. The estimated amplitude of the magnetic anomaly vector did not present remarkable artifacts and striations in the direction of declination. The maximum of the amplitude of the magnetic anomaly vector obtained with equivalent layer technique coincides with the dipolar feature of the observed total-field anomaly in southeast part of the studied region, indicating a possible correspondence with the local geology. In this way, the inversion or the qualitative interpretation of the amplitude of the magnetic anomaly vector can provide a better geologic interpretation in comparison with the inversion or the qualitative interpretation of the total-field anomaly. More tests are required to investigate the limitations of the equivalent layer technique in presence of remanent magnetization.

\section{Acknowledgments}

S.P. Gonzalez was supported by a Phd scholarship from CAPES and F.F. Melo from FAPERJ (grant E26/200.532/2018). V.C.F. Barbosa was supported by fellowships from: CNPQ (grant 307135/2014-4) and FAPERJ (grant E-26/203.091/2016). V.C. Oliveira Junior was supported by fellowships from: CNPQ (grant 308945/2017-4) and FAPERJ (grant E-26/202.729/2018). The authors thank CPRM for permission to use the aeromagnetic data set.

\section{References}

Almeida, J. D. A. C., R., S. B., Dall'Agnol, Dias, and F. J., Althoff, 2010, Origin of the Archean leucogranodioritegranite suites: evidence from the Rio Maria terrane and implications for granite magmatism in the Archean: Lithos, 120, no. 3-4, 235-257.

Aster, R. C., B. Borchers, and C. H. Thurber, 2004, Parameter estimation and inverse problems (international geophysics): Elsevier Academic Press.

Blakely, R., 1995, Potential Theory in Gravity \& Magnetic Application.

CPRM, 2015, Project: Aerogeophysical survey of Carajás: Survey and data processing: Final report, Rio de Janeiro, Brazil, 22 and maps (in Portuguese).
Chulliat, A., S. Macmillan, P. Alken, C. Beggan, M. Nair, B. Hamilton, A. Woods, V. Ridley, S. Maus, and A. Thomson, 2014, The US/UK World Magnetic Model for 2015-2020: NOAA National Geophysical Data Center.

Dampney, C. N. G., 1969, The equivalent source technique: Geophysics, 34, no. 1, 39-53.

Grainger, C. J., D. I. Groves, F. H. Tallarico, and I. R. Fletcher, 2008, Metallogenesis of the Carajás mineral province, southern Amazon craton, Brazil: Varying styles of Archean through Paleoproterozoic to Neoproterozoic base-and precious-metal mineralisation: Ore Geology Reviews, 33, 451-489.

Klein, E. L., J. B. Rodrigues, J. D. S. Queiroz, R. G. Oliveira, S. B. Guimarães, and C. L. Chaves, 2016, Deposition and tectonic setting of the Paleoproterozoic Castelo dos Sonhos metasedimentary formation, Tapajós Gold province, Amazonian Craton, Brazil: age and isotopic constraints: International Geology Review, 59, 121.

Krahenbuhl, R. A., and Y. Li, 2017, Investigation of magnetic inversion methods in highly magnetic environments under strong self-demagnetization effect: Geophysics, 82, no. 6, J83-J97.

Leão-Santos, M., Y. Li, and R. Moraes, 2015, Application of $3 \mathrm{D}$ magnetic amplitude inversion to iron oxide-coppergold deposits at low magnetic latitudes: A case study from Carajás Mineral Province, Brazil: Geophysics, 80, no. 2, B13-B22.

Li, Y., S. E. Shearer, M. M. Haney, and N. Dannemiller, 2010, Comprehensive approaches to $3 D$ inversion of magnetic data affected by remanent magnetization: Geophysics 75, L1-L11.

Pedersen, L. B., 1978, Wavenumber domain expressions for potential fields from arbitrary 2-, 2 1/2 -, and 3dimensional bodies: Geophysics, 43, 626-630.

Silva, J. B. C., 1986, Reduction to the pole as an inverse problem and its application to low-latitude anomalies: Geophysics, 51, no. 2, 369-382.

Siqueira, F. C., V. C., Oliveira Jr, and V. C., Barbosa, 2017, Fast iterative equivalent-layer technique for gravity data processing: A method grounded on excess mass constraint. Geophysics, 82, no. 4, G57-G69.

Tassinari, C. C., and M.J. Macambira, 1999, Geochronological provinces of the Amazonian Craton: Episodes-Newsmagazine of the International Union of Geological Sciences, 22, no.3, 174-182.

Tikhonov, A. N., and V. Y., Arsenin, 1977, Solutions of illposed problems: WH Winston, Washington, DC, 330. 